\title{
Calogero-Bogoyavlenskii-Schiff denkleminin analitik çözümleri
}

\author{
Güldem YILDIZ, ${ }^{1, *}$, Çiğdem TÜRKMEN² \\ ${ }^{1}$ Niğde Ömer Halisdemir Üniversitesi, Fen Edebiyat Fakültesi, Matematik. Böl., Merkez Yerleşke, Niğde \\ ${ }^{2}$ Niğde Ömer Halisdemir Üniversitesi Fen Bilimleri Enstitüsü, Merkez Yerleşke, Niğde
}

Geliş Tarihi (Received Date): 08.10.2020

Kabul Tarihi (Accepted Date): 28.01.2021

\section{Öz}

Kısmi Diferansiyel denklemlerin analitik çözümleri, kuantum mekanik ve plazma fiziği gibi alanlarda, açı̆̆a çıkmamış olayların açıklanmasında fayda sağlamaktadır. Bu çalışmada, lineer olmayan klsmi türevli Calogero-Bogoyavlenskii-Schiff (CBS) diferansiyel denkleminin analitik çözümlerini bulmak için Homojen Denge Metodundan yararlanılmıştır. Homojen Denge Metodunun Calogero-Bogoyavlenskii-Schiff denklemine uygulanmasıyla elde edilen analitik çözümler literatürde bulunan sonuçlarla karşılaştırılmış ve literatürde bulunan çözümlerle uyumlu hiperbolik ve trigonometrik tipten fonksiyonlar elde edilmiştir.

Anahtar kelimeler: Calogero-Bogoyavlenskii-Schiff (CBS) denklemi, homojen denge metodu.

\section{Analytical solutions of Calogero-Bogoyavlenskii-Schiff equation}

\begin{abstract}
Analytical solutions of Partial Differential equations are useful in explaining unrevealed events in areas such as quantum mechanics and plasma physics. In this study, the Homogeneous Balance Method is used to find analytical solutions of the nonlinear partial differential Calogero-Bogoyavlenskii-Schiff (CBS) differential equation. Analytical solutions obtained by applying the Homogeneous Balance Method to the Calogero-Bogoyavlenskii-Schiff equation are compared with the results found in the literature, and hyperbolic and trigonometric type functions that are compatible with the solutions in the literature are obtained.
\end{abstract}

\footnotetext{
*Güldem YILDIZ, guldem.yildiz@ohu.edu.tr, http://orcid.org/0000-0002-8120-3525

Çiğdem TÜRKMEN, cigdemturkmen51@gmail.com, http://orcid.org/0000-0002-8354-8236
} 
Keywords: Calogero-Bogoyavlenskii-Schiff (CBS) equation, homogeneous balance method.

\section{Giriş}

Lineer olmayan kısmi türevli diferansiyel denklemlerden; plazma fiziği [1,2], akışkanlar mekaniği [3], fiber optik [4] ve birçok bilim alanında yararlanılmaktadır. Bu denklemlerin çözümlerini elde etmek için nümerik ve analitik metotlar geliştirilmiştir [5]. Bu metotlardan; Varyasyonel İterasyon Metot [6, 7], Homotopi Analiz Metot [8], Homotopi Pertürbasyon Metot [9-11], Adomian Ayrışım Metot [12], Homojen Denge Metot [13-24] ve diğerleri son yirmi yılda litaratürde bulunan çalışmalardandır. Lineer olmayan denklemlerin çözümlerine Homojen Denge Metodun kullanımıyla hızlı ve analitik sonuçlara ulaşılmaktadır. Lineer olmayan kısmi türevli Korteweg-de Vries denklemlerinden olan Calogero-Bogoyavlenskii-Schiff (CBS) denklemi, farklı çalışmalarla tanıtılmıştır [25-34]. Bu çalışmada, CBS denkleminin analitik çözümlerini elde etmek için yapılan literatür çalışmasında Homojen Denge Metodun bu denkleme uygulanmadığı tespit edilmiştir. Homojen Denge Metodun lineer olmayan denklemlerin analitik çözümlerinin elde edilmesinde uygun bir metot olmasından dolayı CBS denklemine uygulanmıştır.

Lineer olmayan kısmi türevli CBS denklemi, $U$ bağımlı değişken, $x, t$ bağımsız değişkenler olmak üzere;

$U_{x t}+2 U_{y} U_{x x}+4 U_{x} U_{x y}+U_{x x x y}=0$,

şeklindedir.

$(2+1)$ boyutlu CBS (1) denklemi, Riemann'in y ekseni boyunca yayılan dalga ile $\mathrm{x}$ ekseni boyunca yayılan dalga arasındaki etkileşimi açıklamaktadır [25].

\section{Homojen denge metodu}

Lineer olmayan denklemlerin analitik çözümlerini elde etmek için kullanılan Homojen Denge metodu, [13-24] kaynaklarında incelenmiştir. Bu metot, dengeleme terimi esasına dayanır ve kolay bir algoritması vardır. Homojen Denge metodu ile lineer olmayan bir kısmi türevli diferansiyel denklemin tam çözümü,

$U(\mu)=\sum_{i=0}^{M} q_{i} \varphi^{i}(\mu)$

formunda aranır. Burada $q_{i}$ ler sabit ve

$\varphi=\tanh (k \mu), \varphi=\operatorname{coth}(k \mu)$

fonksiyonları da

$\varphi^{\prime}=a \varphi^{2}+b \varphi+c$ ve $\varphi^{\prime}=k\left(1-\varphi^{2}\right)$, 
denklemlerin çözümleridir. Homojen Denge metot, kısaca aşağıdaki maddeler halinde özetlenebilir;

i. Lineer olmayan bir kısmi türevli diferansiyel denklem

$F\left(U, U_{t}, U_{x}, U_{t t}, U_{x t}, U_{x x}, \ldots\right)=0$,

şeklinde yazılabilir. $U=U(x, t)$ bilinmeyen fonksiyon olmak üzere (3) denkleminde

$U(\mu)=U(x, \mathrm{t}), \mu=k x+l t+d$,

dönüşümü uygulanırsa;

$$
P\left(U, U^{\prime}, U^{\prime \prime}, U^{\prime \prime \prime}, \ldots\right)=0
$$

kapalı formda (5) adi diferansiyel denklemi elde edilir.

ii. (3) numaralı denklemin çözümü ise (2) formunda bulabilmek için $N$ dengeleme terimine ihtiyaç vardır. Bu metodun işleyişi "dengeleme terimi" olarak adlandırılan ve en yüksek mertebeli lineer terim ile en yüksek dereceli lineer olmayan terim karşılaştırılmasına dayanır. Bu $N$ dengeleme terimi lineer olmayan herhangi bir adi diferansiyel denklemde en yüksek mertebeden lineer olan terim $\frac{d^{q} u}{d \mu^{q}}$, en yüksek dereceden lineer olmayan terim $u^{p}\left(\frac{d^{r} u}{d \mu^{r}}\right)^{s}$ ve $N$ dengeleme terimi olmak üzere $N+q=N q+s(N+r)$ eşitliğinden elde edilir [23].

iii. Dengeleme terimi $N$ değeri bulunduktan sonra (2) de yerine yazılarak (3) denkleminin çözümü:

$U(\eta)=\alpha_{N}(\varphi)^{N}+\alpha_{N-1}(\varphi)^{N-1}+\ldots+\alpha_{0}=0$

formunda aranır. Burada $\varphi$,

$\varphi^{\prime}=a \varphi^{2}+b \varphi+c$ veya $\varphi^{\prime}=k\left(1-\varphi^{2}\right)$

denklemlerin çözümüdür ve $a, b$,c ve $k$ sabitler olmak üzere $U$ ve türevleri alınarak, mathematica gibi programlar yardımıyla, elde edilen sabitler (5) numaralı denklemde yerlerine yazılır.

iv. (5) numaralı denklemde $U$ ve türevleri yerine yazılırsa, katsayılar $a_{i}$ 'lerden oluşan $\varphi$ 'li cebirsel denklemler elde edilir. Bu cebirsel denklemler düzenlenerek elde edilen $\varphi$ 'nin katsayıları,

$P_{n}\left(q_{m}, q_{m-1}, \ldots, \mathrm{q}_{0} k, w, \ldots\right)=0,(n=0,1,2,3, \ldots, m)$ 
şeklindedir. Bulunan cebirsel denklem sistemleri birtakım hesap programları yardımıyla sıfıra eşitlenerek çözümlenir.

v. Elde edilen çözümler (2) çözüm önerisinde yerine yazılarak (5) numaralı denklemin tam çözümüne ulaşılır. (5) denklemi (3) denkleminin dönüşümü olduğu için (3) numaralı denklemin çözümü elde edilmiş olur [13-24].

\section{Calogero-Bogoyavlenskii-Schiff (CBS) denkleminin homojen denge metodu ile analitik çözümleri}

Lineer olmayan kısmi türevli Calogero-Bogoyavlenskii-Schiff (CBS) (1) numaralı denklemine

$U(x, y, t)=U(\eta), \eta=x+y+\lambda t$,

dönüşümü uygulanırsa,

$U^{\prime \prime \prime}+3\left(\left(U^{\prime}\right)^{2}\right)^{\prime}+\lambda U^{\prime \prime}=0$,

(7) denklemi elde edilir. $c$ integral sabiti olmak üzere, (7) denkleminin integrali alınırsa

$U^{\prime \prime \prime}+3\left(U^{\prime}\right)^{2}+\lambda U^{\prime}+\mathrm{c}=0$

(8) denklemi bulunur. (8) denkleminde $U^{\prime}=V$ alınır ve düzenlenirse,

$$
V^{\prime \prime}+3 V^{2}+\lambda V+\mathrm{c}=0
$$

denklemi elde edilir. (9) denkleminde $V^{\prime \prime}$ ile $V^{2}$ terimleri arasında dengeleme işlemi yapılırsa, dengeleme terimi $m+2=2 m, \quad m=2$ elde edilir. Bu yüzden çözüm önerisi

$V(\eta)=a_{0}+a_{1} \varphi+a_{2} \varphi^{2}$

şeklindedir. Burada,

$\varphi^{\prime}=k \varphi^{2}+l \varphi+m$,

(11) denklemi ve $k, l, m$ sabitler olmak üzere;

$$
\begin{aligned}
V^{\prime} & =a_{1} \varphi^{\prime}=a_{1}\left(k \varphi^{2}+l \varphi+a m\right), \\
V^{\prime \prime} & =2 a_{1} k \varphi \varphi^{\prime}+a_{1} l \varphi^{\prime}=2 a_{1} k \varphi\left(k \varphi^{2}+l \varphi+a m\right)+a_{1} l\left(k \varphi^{2}+l \varphi+a m\right), \\
& =2 a_{1} k^{2} \varphi^{3}+2 a_{1} k l \varphi^{2}+2 a_{1} k m \varphi+a_{1} k l \varphi^{2}+a_{1} l^{2} \varphi+a_{1} l m, \\
& =2 a_{1} k^{2} \varphi^{3}+3 a_{1} k l \varphi^{2}+2 a_{1} k m \varphi+a_{1} l^{2} \varphi+a_{1} l m,
\end{aligned}
$$


$V$ 'nin türevleri bulunur ve (9) denkleminde yerlerine yazılırsa,

$$
\begin{aligned}
& 3 a_{2}^{2} \varphi^{4}+6 a_{2} a_{1} \varphi^{3}+6 a_{2} a_{0} \varphi^{2}+6 a_{2} k^{2} \varphi^{4}+10 a_{2} k l \varphi^{3}+8 a_{2} k m \varphi^{2}+4 a_{2} l^{2} \varphi^{2}+6 a_{2} \operatorname{lm} \varphi+2 a_{2} m^{2} \\
& +a_{2} \varphi^{2} \lambda+3 a_{1}^{2} \varphi^{2}+6 a_{1} a_{0} \varphi+2 a_{2} k^{2} \varphi^{3}+3 a_{1} k l \varphi^{2}+2 a_{1} k m \varphi+a_{1} l^{2} \varphi \\
& +a_{1} l m+a_{1} \varphi \lambda+3 a_{0}^{2}+a_{0} \lambda+c=0
\end{aligned}
$$

denklemi elde edilir. Burada $\varphi$ ve kuvvetleri sıfır olmayacağından $\varphi$ ve kuvvetlerinin katsayılarından oluşan,

$$
\begin{aligned}
& \varphi^{4}: 3 a_{2}\left(a_{2}+2 k^{2}\right)=0, \\
& \varphi^{3}: 2\left(3 a_{2} a_{1}+5 a_{2} k l+a_{1} k^{2}\right)=0, \\
& \varphi^{2}: 6 a_{2} a_{0}+8 a_{2} k m+4 a_{2} l^{2}+a_{2} \lambda+3 a_{1}^{2}+3 a_{1} k l=0, \\
& \varphi^{1}: 6 a_{2} l m+6 a_{1} a_{0}+2 a_{1} k m+a_{1} l^{2}+a_{1} \lambda=0, \\
& \varphi^{0}: 2 a_{2} m^{2}+a_{1} l m+3 a_{0}^{2}+a_{0} \lambda+c=0,
\end{aligned}
$$

denklem sistemi elde edilir. Bu denklemler Mathematica yardımıyla çözümlendikten sonra,

$$
\begin{aligned}
& a_{2}=-2 k^{2}, a_{1}=\frac{12 k^{2} l m}{6 a_{0}+2 k m+l^{2}+\lambda}, a_{0}=\frac{-8 k m-l^{2}-\lambda}{6}, \\
& c=\frac{-16 k^{2} m^{2}+8 k l^{2} m-l^{4}+\lambda^{2}}{12} .
\end{aligned}
$$

katsayıları elde edilir.

Durum 1: (11) denklemi için $\varphi^{\prime}$ ve $\varphi^{2}$ terimleri arasında dengeleme terimi hesaplanırsa dengeleme teriminin değeri $m+1=2 m, m=1$ elde edilir. Buradan Ricattti denkleminin özel çözümü olan $\varphi=\sum_{i=0}^{m} b_{i} \tanh ^{i} \eta$ den çözüm önerisi,

$$
\varphi=b_{0}+b_{1} \tanh (\eta)
$$

elde edilir. (13) denklemi (11) denkleminde yerine yazılırsa,

$$
-b_{1} \tanh (\eta)^{2} k-2 b_{0} b_{0} \tanh (\eta) k-b_{1} \tanh (\eta)^{2}-b_{1} \tanh (\eta) 1+b_{1}-b_{0}^{2} k-b_{0} l-m=0
$$

denklemi elde edilir. Bu denklem düzenlendiğinde,

$$
-b_{1}\left(b_{1} k+l\right)[\tanh (\eta)]^{2}=0
$$


$b_{1}\left(-2 b_{0} k-l\right) \tanh (\eta)=0$,

$b_{1}-b_{0}^{2} k-b_{0} l-m=0$

denklem sistemine ulaşılır. Bu denklem sisteminin çözümleri,

$b_{1}=\frac{-1}{k}, b_{0}=\frac{-l}{2 k}, m=\frac{l^{2}-4}{4 k}$,

şeklindedir. (12) ve (14) denklemleri (10) ve (13) denklemlerinde yazılarak düzenlenirse (9) denklemin çözümü,

$V=\frac{-12 \tanh (\eta)^{2}-\lambda+8}{6}$

şeklindedir ve (15) numaralı denklemde $U^{\prime}=V$ alınır ve düzenlenirse, (1) denklemin çözümü,

$U_{1}(\eta)=-\frac{1}{6} \eta(4+\lambda)+2 \tanh \eta$

(16) hiperbolik tip fonksiyon şeklinde elde edilir.

Benzer şekilde aynı işlemler $\varphi=\sum_{i=0}^{m} b_{i} \operatorname{coth}^{i} \eta$ içinde yapılırsa,

$V(\eta)=\frac{-12 a_{1} \operatorname{coth}(\eta) b-\lambda+8}{6}$,

denklemi elde edilir. (17) denkleminde $U^{\prime}=V$ alınır ve düzenlenirse, (1) denklemin çözümü,

$U_{2}(\eta)=-\frac{1}{6} \eta(4+\lambda)+2 \operatorname{coth} \eta$,

şeklinde olur. (16) ve (18) çözümleri; birbirleriyle benzer hiperbolik tip fonksiyonlardır.

Durum 2: (11) denkleminde $k=1, l=0$ için $\varphi^{\prime}=\varphi^{2}+m$ olur ve

$\varphi(\eta)=\left\{\begin{array}{cc}-\sqrt{-m} \tanh (\sqrt{-m} \eta), & m<0 \\ -\frac{1}{\eta}, & m=0 \\ \sqrt{m} \tan (\sqrt{m} \eta), & m>0\end{array}\right.$

çözümleri elde edilir. Buradan da (1) denklemin çözümü, $m<0$ için, 
$V(\eta)=\frac{12 \tanh (\sqrt{-m} \eta)^{2} \mathrm{~m}-8 m-\lambda}{6}$

$U^{\prime}=V$ alınır ve düzenlenirse,

$U_{3}(\eta)=\frac{1}{6} \eta(4 m-\lambda)+2 \sqrt{-m} \tanh \sqrt{-m} \eta$,

$m=0$ için,

$V(\eta)=\frac{-\eta^{2} \lambda-12}{6 \eta^{2}}$

$U^{\prime}=V$ alınır düzenlenirse,

$U_{4}(\eta)=\frac{2}{\eta}-\frac{\eta \lambda}{6}$

şeklinde hiperbolik tip çözümler elde edilir ve $m>0$ için,

$V(\eta)=\frac{-12 \tan (\sqrt{m} \eta)^{2} \mathrm{~m}-8 \mathrm{~m}-\lambda}{6}$

$U^{\prime}=V$ alınır ve düzenlenirse,

$U_{5}(\eta)=\frac{1}{6} \eta(4 m-\lambda)-2 \sqrt{m} \tan \sqrt{m} \eta$,

şeklinde trigonometrik tip çözüm elde edilir.

(19) fonksiyonunun $m=-1, \lambda=1$ değerleri için

$U(\eta)=-\frac{5}{6} \eta+2 \tanh \eta$,

Çözüm eğrisinin grafiği Şekil 1 de gösterilmiştir.

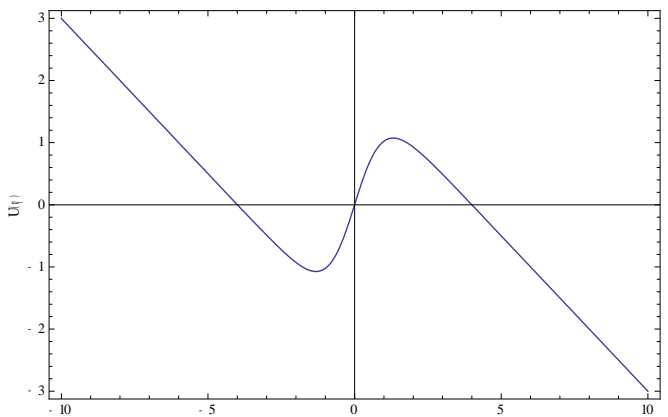

Şekil 1. CBS denkleminin, $U(\eta)=-\frac{5}{6} \eta+2 \tanh \eta$, çözüm eğrisi. 


\section{Sonuçlar}

$\mathrm{Bu}$ çalışmada, lineer olmayan dalga denklemlerinden Calogero-Bogoyavlenskii-Schiff CBS denklemine Homojen Denge metodu uygulanmıştır. Bulunan fonksiyonlar ve türevleri CBS denkleminde yerine yazılarak sonuçların doğruluğu tespit edilmiştir. Bu metotla CBS denkleminin hiperbolik ve trigonometrik tipte çözümlerine ulaşılmıştır. Yukarıda ifade edilen bu çözümler [31] kaynağında bulunan çözümlerle uyumludur. Ancak, [31] kaynağında Sine-Gordon Açılım Metodundan faydalanarak kompleks fonksiyon çözümleri de elde edilmiştir. Bu sebeple, lineer olmayan kısmi türevli denklemlerin hiperbolik ve trigonometrik tipte çözümlerini elde etmek için kolay algoritmasından dolayı Homojen Denge metodu tercih edilebilir.

\section{Kaynaklar}

[1] Goldston, R. J. ve Rutherford, P. H., Introduction to Plasma Physics, CRC Press, (1995).

[2] Dönmez, O. ve Dağhan, D., Analytic Solutions of the Schamel-KdV Equation by Using Different Methods: Application to a Dusty Space Plasma, Süleyman Demirel Üniversitesi Fen Bilimleri Enstitüsü Dergisi, 21, 208-215, (2017).

[3] Çengel, Y. A. ve Cımbala J. M., Akışkanlar Mekaniği, Güven Bilimsel, (2008).

[4] Anderson, D., Variational approach to nonlinear pulse propagation in fibers, Physics Letters A, 27, 3135-3145, (1983).

[5] Yavuz, M. ve Yokus A., Analytical and numerical approaches to nerve impulse model fractional - order, Numerical Methods for Partial Differential Equations, 36(6), 1348-1368, (2020).

[6] Evirgen, F., Analyze the optimal solutions of optimization problems by means of fractional gradient based system using VIM, An International Journal of Optimization and Control: Theories \& Applications (IJOCTA), 6, 75-83, (2016).

[7] Evirgen, F. ve Yavuz M., An Alternative Approach for Nonlinear Optimization Problem with Caputo - Fabrizio Derivative, ITM Web of Conferences 22, 01009, (2018).

[8] Yavuz, M. ve Yaşkıran B., Homotopy methods for fractional linear/nonlinear differential equations with a local derivative operator, Journal of Balıkesir University Institute of Science and Technology, 20(3) Special Issue, 75-89, (2018).

[9] Y1ldız, G. ve Dağhan D., Solution of the (2+1) Dimensional Breaking Soliton Equation by Using Two Different Methods, Journal of Engineering Technology and Applied Sciences, 1, 13-18, (2016).

[10] Dağhan D., Yavuz H. ve Yıldız G., Lineer Olmayan Kısmi Türevli Denklemlere Homotopi Pertürbasyon Yönteminin Uygulanmas1, Ömer Halisdemir Üniversitesi Mühendislik Bilimleri Dergisi, 6, 290-301, (2017).

[11] Yavuz M. ve Ozdemir N., Numerical Inverse Laplace Homotopy Technique for Fractional Heat Equations, Thermal Science, 22, 185-194, (2018).

[12] Yavuz M., Novel solution methods for initial boundary value problems of fractional order with conformable differentiation, An International Journal of Optimization and Control: Theories \& Applications, 8, 1-7, (2018). 
[13] Wang, M., Zhou, Y., ve Li, Z., Application of a homogeneous balance method to exact solutions of nonlinear equations in mathematical physics, Physics Letters A, 216, 67-75, (1996).

[14] Fan, E. ve Zhang, H., A note on the homogeneous balance method, Physics Letters A, 246, 403-406, (1998).

[15] Fan, E., Two new applications of the homogeneous balance method, Physics Letters A, 265, 353-357, (2000).

[16] Senthilvelan, M., On the extended applications of Homogenous Balance method, Applied Mathematics and Computation, 123, 381-388, (2001).

[17] Zhao, X. ve Tang, D., A new note on a homogeneous balance method, Physics Letters A, 297, 59-67, (2002).

[18] Zhao, X., Wang, Li. ve Sun W., The repeated homogeneous balance method and its applications to nonlinear partial differential equations, Chaos, Solitons and Fractals, 28, 448-453, (2006).

[19] Jie, J., Wu, J. ve Zhang, J., Homogeneous Balance method for an Inhomogeneous $\mathrm{KdV}$ equation: Böcklund Transformation and Lax Pair, International Journal of Nonlinear Science, 9, 69-71, (2010).

[20] Abdel Rady, A.S., Osman, E.S., ve Khalfallah, M., The homogeneous balance method and its application to the Benjamin-Bona-Mahoney (BBM) equation, Applied Mathematics and Computation, 217, 1385-1390, (2010).

[21] Zayed, M.E., ve Alurrfi, K.A.E., The Homogeneous Balance method and its applications for nonlinear evolution equations, Italian Journal of the Applied Mathematics, 33, 307-318, (2014).

[22] Yi, W., He, X-D. ve Yang X-F., The homogeneous balance of undetermined coefficients method and its application, Open Mathematics, 14, 816-826, (2016).

[23] Türkmen, Ç., Lineer Olmayan Kısmi Türevli Diferansiyel Denklemlere Kudryashov Metodu ve Homojen Denge Metodunun Uygulanması, Yüksek Lisans Tezi, Niğde Ömer Halisdemir Üniversitesi, (2019).

[24] Radha, B. ve Duraisamy, C., The homogeneous balance method and applications for finding the exact solutions for nonlinear equations, Journal of Ambient Intelligence and Humanized Computing, (2020).

[25] Yu, SJ., Toda, K.ve Fukuyama T., N-soliton solutions to a (2+1)-dimensional integrable equation, Journal of Physics A: Mathematical and General, 31, 10181-10186, (1998).

[26] Bruzon, M.S., Gandarias, M.L., Muriel, C., Ramierez, J., Saez, S. ve Romero, F.R., The Calogero-Bogoyavlenskii-Schiff equation in $2+1$ dimensions, Theoretical and Mathematical Physics, 137 (1), 1367-1377, (2003).

[27] Kobayashi, T. ve Toda K., The Painlevé test and reducibility to the canonical forms for higher-dimensional soliton equations with variable-coefficients, Symmetry, Integrability and Geometry: Methods and Applications, 2, 1-10, (2006).

[28] Peng, Y., New types of localized coherent structures in the BogoyavlenskiiSchiff equation, International Journal of Theoretical Physics, 45 (9), 17791783, (2006).

[29] Wazwaz, A.M., New solutions of distinct physical structures to highdimensional nonlinear evolution equations, Applied Mathematics and Computation, 196, 363-370, (2008). 
[30] Kaplan, M., Bekir, A. ve Akbulut, A., A generalized Kudryashov method to some nonlinear are evolution equations in mathematical physics, Nonlinear Dynamics, 85, 2843-2850, (2016).

[31] Başkonuş, H. M., Sulaiman, T. A. ve Bulut, H., New solitary wave solutions to the $(2+1)$ dimensional Calogero-Bogoyavlenskii-Schiff and the Kadomtsev Petviashvilihierarchy equations, Indian Journal of Physics, 91, 1237-1243, (2017).

[32] Tahami, M. ve Najafi, M., Multi-wave solutions for the generalized (2+1)dimensional nonlinear evolution equations, Optik, 136, 228-236, (2017).

[33] Salem, S., Kassem, M., Mohamed ve Mabrouk, S. M., Similarity Solution of (2+1)Dimensional Calogero-Bogoyavlenskii-Schiff Equation Lax Pair, American Journal of Applied Mathematics, 7 (5), 137-144, (2019).

[34] Saleh, R., Kassem, M. ve Mabrouk, S. M., Investigation of breaking dynamics for Riemann waves in shallow water, Chaos, Solitons and Fractals, 132, 109571, (2020). 